\title{
DISTRIBUCION DE ELEMENTOS MECÁNICOS EN PILAS SUJETAS A FUERZAS EN LA CABEZA Y ANTE LA INCIDENCIA DE ONDAS SÍSMICAS
}

\author{
Luciano R Fernández Sola ${ }^{(1)}$, Javier Avilés López ${ }^{(2)}$ y David Muriá Vila ${ }^{(3)}$
}

\begin{abstract}
RESUMEN
El diseño convencional de pilas y pilotes ante cargas laterales considera de manera casi exclusiva la acción de las fuerzas que induce la estructura en su cabeza. El paso de las ondas sísmicas a través de los elementos de cimentación también produce solicitaciones mecánicas debidas a la incompatibilidad de deformaciones entre el suelo y la pila o el pilote. La distribución y magnitud de los elementos mecánicos debidos a ambos fenómenos difiere en gran medida, y depende de la estratigrafía y las condiciones de apoyo de la pila o pilote. En este trabajo se utiliza un método de estratos finitos para estimar los elementos mecánicos en pilas y pilotes ante cargas dinámicas. Se considera la restricción parcial al giro en la punta del elemento de cimentación y la conexión de la cabeza con la superestructura. Estos aspectos tienen influencia en la respuesta lateral de pilas y pilotes, sobre todo para elementos de gran diámetro. Se desarrolla un análisis paramétrico para determinar las diferencias cualitativas entre los elementos mecánicos introducidos por la aplicación de fuerzas en la cabeza de la pila o pilote y aquellos generados por el paso de ondas sísmicas.
\end{abstract}

Palabras Clave: Pilas y pilotes; interacción cinemática e inercial; diseño de cimentaciones

\begin{abstract}
Conventional design of piles under lateral loads considers almost in an exclusively way the action of the forces that the structure introduces into the head of the pile. Seismic waves passing through the foundation produces inner forces on the piles too, due to the strain incompatibility between the pile and the surrounding soil. The distribution and magnitude of internal forces due to forces at pile head and wave passage are very different and depend strongly on soil stratigraphy and supporting conditions on the pile toe and head. In this work a finite layer method to compute internal forces generated by lateral solicitations on embedded piles is used. A partial rotation restriction at the pile toe and the connection of the pile to the superstructure is explicitly considered. These issues have an important influence, particularly on piles with large diameters. A parametric analysis for different soil-pile configurations subjected to lateral forces is developed, in order to establish qualitative differences between the solicitations produced by forces at pile head and those produce by wave passage.
\end{abstract}

Key Words: Piles; kinematic and inertial interaction; foundation design

Artículo recibido el 11 de noviembre de 2011 y aprobado para su publicación el 28 de marzo de 2012

(1) Profesor, Departamento de Materiales, Universidad Autónoma Metropolitana Azcapotzalco, Av. San Pablo 180, Col Reynosa Tamaulipas, 02200 México DF, email: lrfs@correo.azc.uam.mx

(2) Investigador, Instituto Mexicano de Tecnología del Agua, Jiutepec 62550, Morelos, javiles@tlaloc.imta.mx

(3) Investigador, Instituto de Ingeniería-UNAM, Ciudad Universitaria, 04510, México D.F., email: dmv@pumas.ii.unam.mx 


\section{INTRODUCCIÓN}

Dentro del comportamiento sísmico de las edificaciones, el desempeño estructural de las cimentaciones tiene un papel preponderante. El análisis y diseño estructural de la subestructura son temas que en muchas ocasiones no reciben la atención adecuada, debido a que requiere una profunda comprensión de dos disciplinas de la ingeniería civil, diseño estructural y geotecnia, las cuales generalmente se estudian por separado.

El diseño sísmico convencional de cimentaciones a base de pilas y pilotes, considera casi de manera exclusiva los elementos mecánicos introducidos por las fuerzas de inercia, desarrolladas por la estructura y aplicadas en la cabeza de la pila o pilote. Dichos elementos mecánicos solamente se presentan en la parte superior de la pila o pilote hasta una profundidad determinada por la longitud activa de la misma, bajo la consideración de que ésta solamente se puede flexionar hasta cierta profundidad, después de la cual se supone empotrada. Este fenómeno se asocia con la parte inercial del fenómeno de interacción dinámica suelo estructura (IDSE).

La IDSE consiste en un conjunto de efectos no solo cinemáticos sino también inerciales producidos en la estructura y el suelo como resultado de la flexibilidad de éste ante excitación sísmica. La interacción modifica las propiedades dinámicas relevantes de la estructura (interacción inercial) así como las características del movimiento del terreno en la vecindad de la cimentación (interacción cinemática). Las fuerzas que desarrolla la estructura sobre las pilas, dependen básicamente de las propiedades dinámicas del edificio. Es por ello que los elementos mecánicos introducidos por fuerzas en la cabeza de las pilas se asocian con esta parte del fenómeno (interacción inercial).

Sin embargo, existen evidencias de daño en pilas y pilotes a profundidades mayores a la longitud activa, por ejemplo en Japón, como lo reporta Mizuno (1987). Este daño ha sido asociado a la interacción cinemática, ya que la incompatibilidad de deformaciones entre la pila y el terreno produce un movimiento diferencial entre los medios, generando rotaciones flexionantes importantes en el elemento de cimentación.

El fenómeno ha sido previamente estudiado en el comportamiento de estructuras subterráneas. Mediciones acerca del comportamiento sísmico de un túnel realizadas por Okamoto et al (1973) muestran que la respuesta del túnel se encuentra dominada por la respuesta del medio que lo rodea y no por las propiedades inerciales de la estructura en sí. Es por esto que el punto medular del diseño de estructuras subterráneas bajo acciones sísmicas, es el campo de desplazamientos del medio y su interacción con la estructura. Este acercamiento es completamente distinto al análisis de las estructuras superficiales, en donde las propiedades inerciales de la estructura son las que controlan en general la respuesta del sistema (Hashash et al 2001).

Para el análisis de pilas el fenómeno es más complicado, ya que la respuesta del elemento y las fuerzas que se desarrollan en éste, se define por dos acciones principalmente. Por un lado, el comportamiento de la pila ante las descargas que recibe de la superestructura. Por otra parte, la influencia del movimiento propio del terreno en la respuesta del elemento de cimentación, tal como se hace para estructuras subterráneas de mayor dimensión.

Es importante entonces determinar cuáles son las principales diferencias entre las distribuciones de los elementos mecánicos dentro de las pilas que se producen por el paso de ondas sísmicas en relación a las distribuciones que se presentan cuando las pilas se someten a cargas en su cabeza. Esta consideración es la más comúnmente utilizada en el diseño convencional. 
Varios autores han estudiado el problema de las fuerzas introducidas en las pilas por acciones aplicadas en la cabeza, y más recientemente, la distribución de los elementos mecánicos debidos a la interacción cinemática. Muchos de los modelos que se han utilizado presentan algunas simplificaciones. Por ejemplo el amortiguamiento del material del que está constituida la cimentación no se toma en cuenta explícitamente.

La mayoría de los métodos simplificados utilizados para analizar el comportamiento de pilas ante cargas laterales, las modelan como vigas de Euler-Bernoulli en las cuáles sólo se considera la rigidez a flexión de la pila $E_{0} I_{0}$ ( $I_{0}$ es el momento de inercia de la sección transversal y $E_{0}$ es el módulo de elasticidad del material con el que está constituida la pila), y su longitud. Por otro lado, estos métodos consideran que no existe restricción al giro en la punta de la pila (apoyo articulado). Una revisión de algunos de los métodos en la literatura se presenta en Fernández (2011).

Esta manera de modelar el problema es válida para elementos con relación de esbeltez alta y con diámetro pequeño, ya que para estos casos efectivamente el comportamiento lateral de la pila queda regido únicamente por la rigidez a flexión, y la punta de la pila no está sujeta a restricciones importantes al giro debido a que el área de apoyo de la misma es muy pequeña, por lo que dicha restricción es despreciable.

Sin embargo, cuando se trata de pilas con diámetro grande y relación de esbeltez baja, su representación por medio de una viga Euler-Bernoulli ya no es adecuada, debido principalmente a que la contribución de la deformación por cortante al comportamiento lateral es importante y que la restricción al giro de la punta debida al diámetro de la pila y la rigidez del suelo de desplante deja de ser despreciable. Se ha observado en pruebas experimentales (Nikolau et al 2001) que la punta de la pila efectivamente desarrolla momento flexionante.

Es entonces recomendable analizar el sistema mediante un método de interacción dinámica que considere adecuadamente el contraste de rigidez entre la pila y el medio, y de manera explícita las condiciones de frontera y la difracción de las ondas producida por la presencia de un elemento de mayor rigidez.

En este trabajo se utilizó la metodología presentada por Fernández et al $(2011,2012)$ que ha sido utilizada también para el análisis sísmico de lumbreras por Avilés y Pérez Rocha (2011).

\section{DESCRIPCIÓN DE LA METODOLOGÍA UTILIZADA}

La metodología utilizada consiste en un modelo basado en estratos finitos (Lysmer y Drake 1972). Permite considerar sin distinción, si el elemento es una pila o un pilote, ya que la formulación del problema aquí utilizada considera explícitamente la influencia del tamaño del diámetro en el comportamiento lateral de la pila o pilote. Es por ello que se refiera a pilas de manera genérica de aquí en adelante.

Se basa en la modelación de una configuración estratigráfica en la cual se definen dos zonas con estratos horizontales que flanquean una zona central en la que el medio puede tener una forma arbitraria (Lysmer y Drake 1972). Estas tres zonas comprenden dos regiones del problema, una correspondiente a las zonas laterales (región exterior) y otra que comprende la zona central (región interior) (figura 1). Entre ellas existen superficies de contacto verticales. Esta metodología puede considerarse como una variante la técnica de los elementos de frontera (Hall, 1994), ya que las ecuaciones de desplazamiento y esfuerzos se evalúan exclusivamente en las fronteras entre las regiones, a diferencia de las metodologías basadas en 
elementos finitos (Zienkiewicz, 1994) en las que se deben evaluar los desplazamientos y esfuerzos para todos los elementos en que se divide el dominio.

Si se considera la región interior como una región axisimétrica estratificada, lo cual para el caso de pilas es una aproximación adecuada, de igual manera que la región exterior (Tassoulas 1981, Tassoulas y Kausel 1983) es posible expresar el campo de desplazamientos en todo el dominio del problema mediante la combinación lineal de modos naturales de propagación en los casos antiplano y plano. Como es bien sabido, en una estructura plana es posible desacoplar el movimiento en el plano del movimiento perpendicular al plano, por lo que el análisis puede ser separado en dos partes: tipo ondas de Love, en el cual todos los movimientos son perpendiculares al plano de la estructura y tipo ondas de Rayleigh en el cual los movimientos son en el plano de la estructura.

Tanto la pila como el medio de desplante se discretizan sólo en la dirección vertical en un número dado de estratos finitos, considerando una variación lineal de los desplazamientos definidos en los nodos extremos de cada estrato. Las ecuaciones diferenciales de movimiento en cada nodo se resuelven mediante el uso de funciones de Bessel. Estas funciones tienen la particularidad de que por su forma satisfacen la condición de irradiación al infinito. Estableciendo las condiciones de frontera pertinentes, en este caso transmisión perfecta de esfuerzos y desplazamientos entre el medio y la pila, se puede definir una ecuación por cada grado de libertad en cada uno de los nodos.

Cada uno de los estratos se encuentra definido por sus propiedades mecánicas al igual que el material de la pila y ( $\beta_{0}$ y $\beta_{j}$ = velocidad de propagación de ondas de cortante del material de la pila y del estrato $j$-ésimo, $\rho_{0}$ y $\rho_{j}=$ densidad de masa del material de la pila y del estrato $j$-ésimo, $\zeta_{0}$ y $\zeta_{j}=$ amortiguamiento del material de la pila y del estrato j-ésimo y $v_{0}$ y $v_{j}=$ relación de Poisson del material de la pila y del estrato $j$-ésimo). Además la pila se encuentra definida por su radio $\left(r_{0}\right)$ y su longitud ( $L$ ), mientras que la formación de suelo está definida por su espesor total ( $H_{s}$ ) (figura 1). De esta manera la metodología considera explícitamente el diámetro, el área de la sección transversal y el amortiguamiento del material de la pila, a diferencia de los métodos simplificados.

Así se establecen las ecuaciones diferenciales homogéneas para ambas regiones, interior y exterior, de las cual se obtienen las formas modales correspondientes para satisfacer las condiciones de frontera.

Para introducir el movimiento basta con encontrar dos soluciones particulares de la ecuación de movimiento, que representen los movimientos de "campo libre" de ambas regiones bajo la acción de un movimiento impuesto en la base del modelo o en la cabeza de la pila, suponiendo que son infinitas en la dirección horizontal, cada una por separado.

Esta metodología también permite conocer el estado de esfuerzos que se genera en el sistema, mediante la estimación de las fuerzas nodales. Además, a diferencia de los métodos simplificados más usados, el modelo considera la aportación al comportamiento lateral de la deformación por cortante de la pila y la contribución del componente vertical del movimiento debido a la rotación del elemento.

Para analizar pilas con cabeza libre y cabeza fija, adicionalmente es necesario introducir una condición aproximada para la restricción del movimiento de la cabeza. En este modelo se utiliza un resorte vertical distribuido a lo largo del perímetro de la cabeza (Fernández et al. 2011). La rigidez de dicho resorte puede ser modificada para considerar distintos niveles de rotación de la cabeza. Cuando se analizan pilas con cabeza libre, el resorte se considera con rigidez cero. Para el caso de pilas con cabeza 
fija, se considera un valor infinito de la rigidez del resorte. En este trabajo se presentan resultados solamente para pilas con cabeza fija.

Dentro de las limitaciones del modelo utilizado se encuentra que, al referirse a una solución en el dominio de la frecuencia, no es posible considerar modelos constitutivos no lineales de los materiales ni de las condiciones de frontera. Una adecuada representación del comportamiento no lineal del suelo puede realizarse mediante la consideración de valores de sus propiedades compatibles con los niveles de deformación esperados.

El desarrollo numérico completo del modelo y la implementación del mismo se puede encontrar en Fernández et al (2011, 2012).

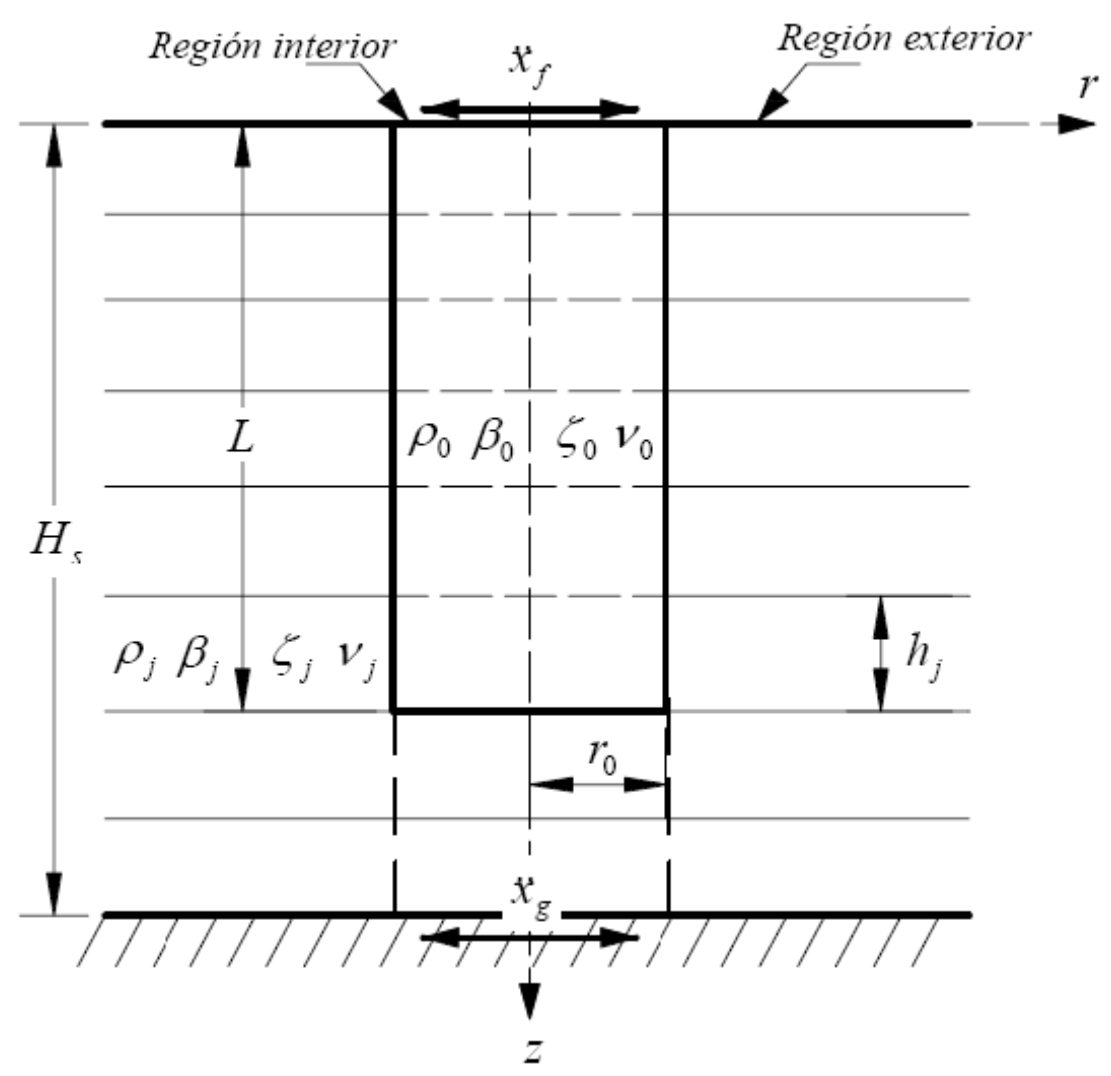

Figura 1. Esquema del modelo

\section{VALIDACIÓN Y COMPARACIÓN CON OTROS AUTORES}

Para validar la metodología propuesta se buscaron resultados de modelos que consideraran hipótesis similares a las que se introducen en el trabajo aquí presentado. Dos tipos de comparaciones se desarrollaron, una que considera los resultados del modelo cuando la excitación se introduce en la base del sistema y otra para validar los resultados del modelo cuando la excitación se introduce en la cabeza de la pila. 
No se encontraron modelos publicados que introdujeran las mismas hipótesis que el presente trabajo, por lo que se decidió validar el modelo reproduciendo resultados de modelos más simplificados, en los cuales las inclusiones se consideran rígidas.

Posteriormente, se compararon los resultados obtenidos con otros modelos ya sea ampliamente utilizados, o de reciente publicación, y se establecen las diferencias entre los resultados de este trabajo y esos modelos, determinando las razones probables de dichas variaciones.

En primera instancia, se reproducen los resultados desarrollados por Veletsos y Younan en 1995, los cuales muestran la respuesta de un cilindro rígido embebido en un medio homogéneo representado por un número de estratos delgados. El cilindro se encuentra desplantado sobre una base infinitamente rígida en la cual se impone el movimiento. Con esta metodología calculan la variación de la fuerza cortante y el momento flexionante en la base con respecto a la relación de esbeltez $H_{s} / r_{0}$. El cortante y el momento están normalizados con $\pi r_{0} \rho_{0} \ddot{x}_{g} H_{s}^{2}$ y $\pi r_{0} \rho_{0} \ddot{x}_{g} H_{s}^{3}$ respectivamente.
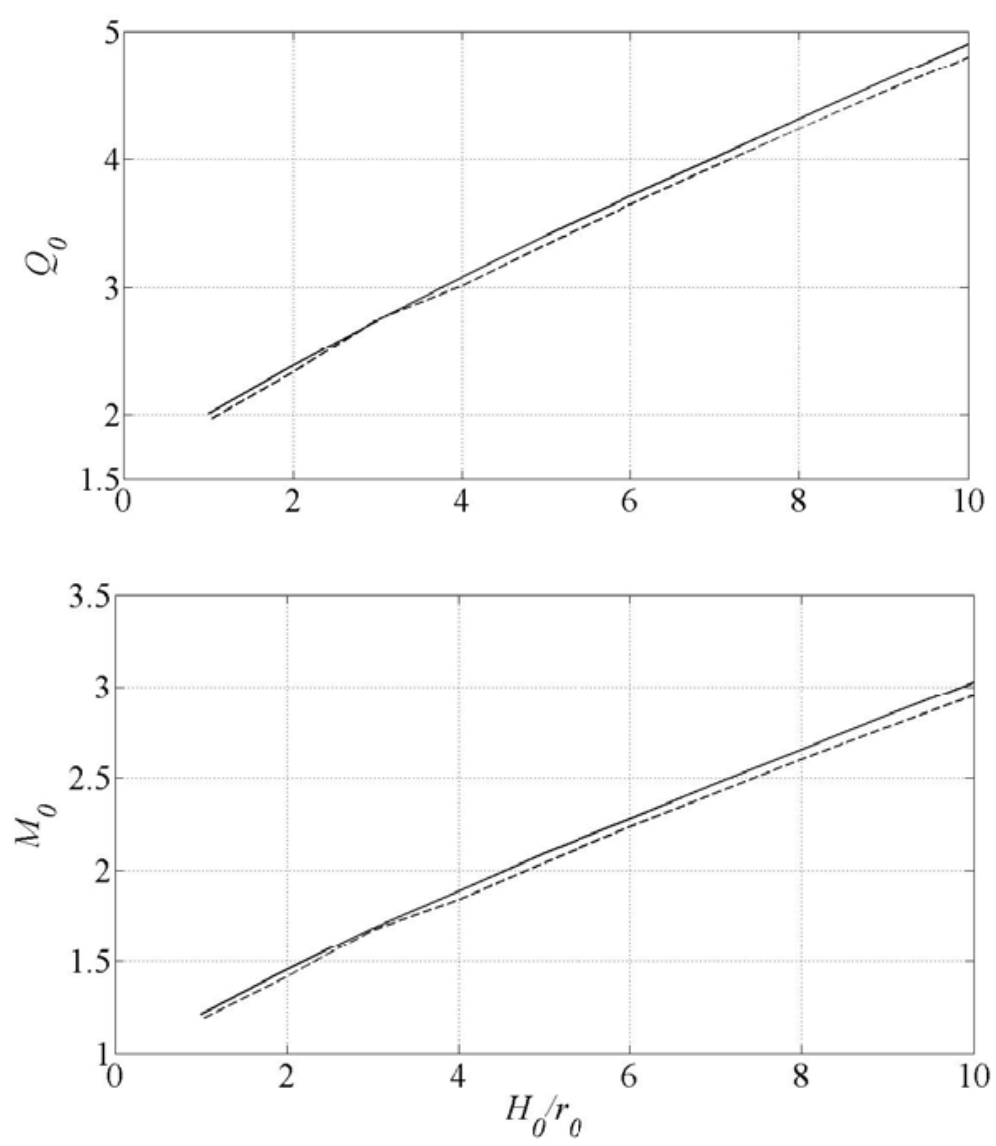

Figura 2. Cortante basal y momento basal para una inclusión rígida con el método de Veletsos y Younan (línea continua) y la metodología propuesta (línea punteada)

Para reproducir la condición de la inclusión infinitamente rígida, se modeló la pila compuesta de un material con velocidad de ondas de cortante mucho mayor a la velocidad de ondas de cortante del suelo circundante. 
En la figura 2 se presentan las gráficas de variación de cortante basal y momento basal de Veletsos y Younan (1995) y las calculadas con el método propuesto respectivamente. Se muestra que los resultados se reproducen aceptablemente con el método propuesto y con las condiciones previamente establecidas.

También se compararon los resultados que presenta Nikolau et al (2001). Este modelo representa a la pila por medio de una viga de Winkler en la cual solamente influyen el módulo de elasticidad $E_{0}$ y el momento de inercia de la sección transversal $I_{0}$.

La pila se encuentra conectada con el suelo circundante por medio de resortes que representan las rigideces horizontales del terreno, los cuales no se encuentran acoplados entre sí, considerando entonces que el desplazamiento a una profundidad específica en el suelo no influye de ninguna manera el desplazamiento en las demás profundidades.

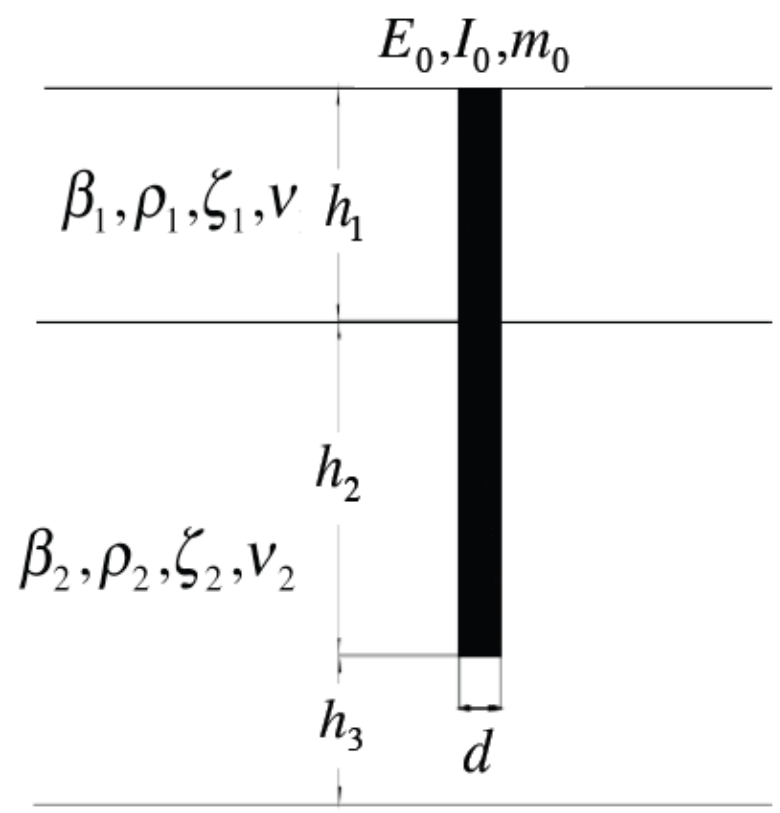

Figura 3. Parámetros del modelo

Nikolau et al (2001) analizan un modelo que contiene los parámetros que se muestran en la figura 3. Se analiza la respuesta estacionaria $\left(\omega=\omega_{1}\right)$ de una pila individual que descansa en un medio compuesto de dos estratos sobre una base rígida. Se comparan tres casos de estudio con los parámetros dados en la tabla 1.

La comparación de resultados con ambas metodologías se muestra en la figura 4. Se puede apreciar que los resultados concuerdan aceptablemente en la mayor parte de la pila. Las diferencias radican principalmente al nivel de la punta; por una parte los resultados de Nikolau et al (2001) son cero, mientras que los resultados determinados con la metodología propuesta presentan valores diferentes a cero en ese mismo punto. 
Tabla 1 Parámetros de las pruebas desarrolladas por Nikolau et al (2001)

\begin{tabular}{ccccccccc}
\hline Prueba & $E_{0} / E_{s}$ & $h_{3} / L$ & $\zeta_{1}=\zeta_{2}$ & $v_{1}=v_{2}$ & $L / d$ & $h_{1} / L$ & $\beta_{1} / \beta_{2}$ & $\rho_{1} / \rho_{2}$ \\
\hline 1 & 5,000 & 1.0 & 0.1 & 0.4 & 20 & $1 / 2$ & $1 / 2$ & 0.8 \\
2 & 5,000 & 1.0 & 0.1 & 0.4 & 20 & $1 / 2$ & $1 / 4$ & 0.7 \\
3 & 5,000 & 1.0 & 0.1 & 0.4 & 20 & $1 / 2$ & $1 / 10$ & 0.6 \\
\hline
\end{tabular}
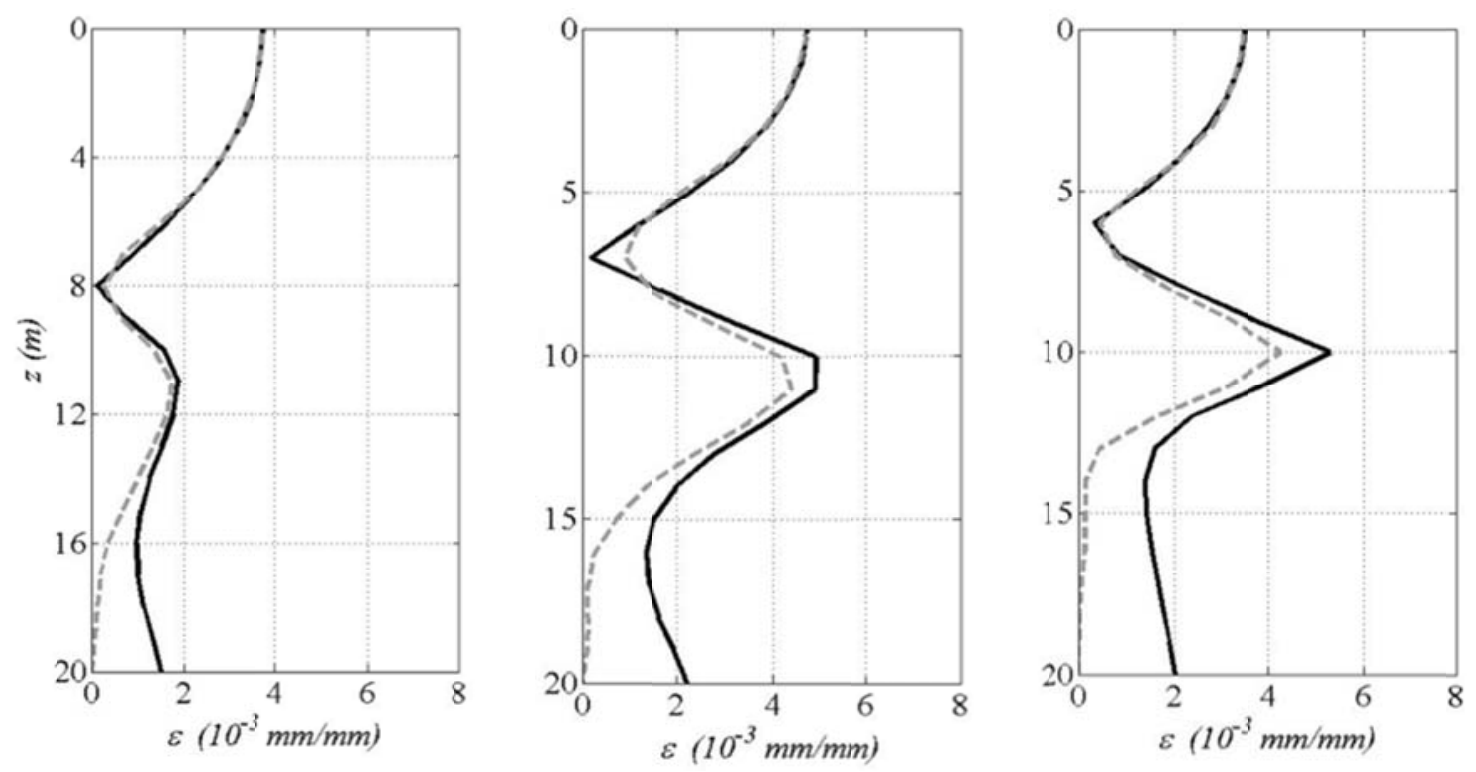

Figura 4. Deformaciones unitarias axiales calculadas con el modelo basado en viga de Winkler (línea punteada) y el modelo propuesto (línea continua).

Esta diferencia se debe a que el modelo basado en una viga de Winkler utilizado por Nikolau et al (2001), considera que la punta de la pila está articulada, en tanto que la metodología propuesta considera explícitamente la restricción ante rotación que se produce debido a que la pila tiene un espesor específico y el suelo de soporte cuenta con una rigidez dada.

En los resultados experimentales que presentan Nikolau et al (2001) para dos pilas dentro de un mismo arreglo, se ve que efectivamente al nivel de la punta, la deformación unitaria axial debida al momento flexionante es diferente de cero, como se muestra en la figura 5.

Por otra parte, se compararon los resultados obtenidos con la metodología propuesta con los de otros autores considerando que ahora el movimiento se impone en la cabeza del elemento de cimentación, para pilas con cabeza fija. Éste tipo de análisis se traduce en el cálculo de las funciones de impedancia horizontales de las cimentaciones. 


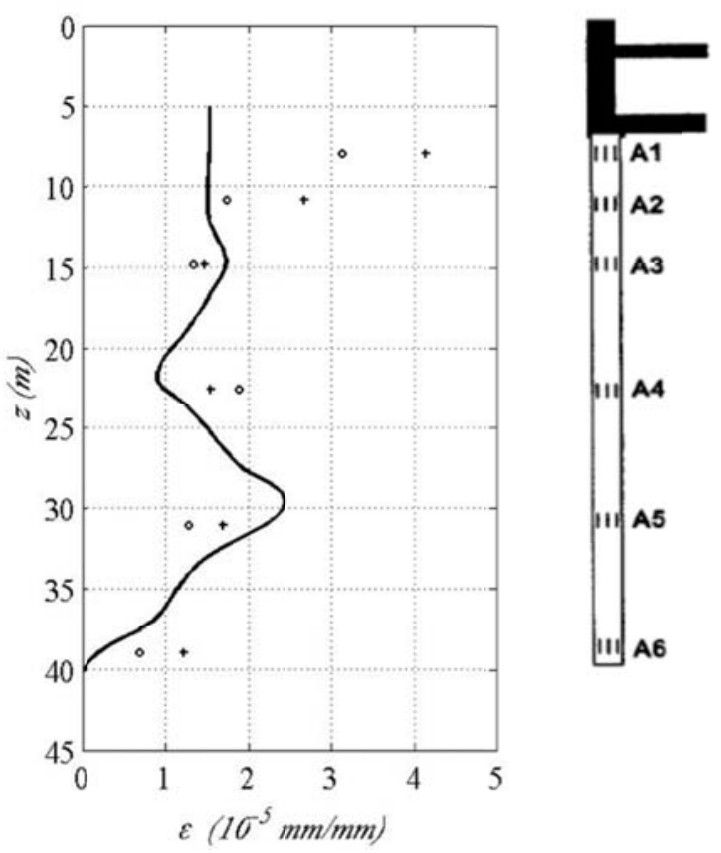

Figura 5. Deformaciones axiales experimentales de dos pilas presentadas por Nikolau et al (2001)
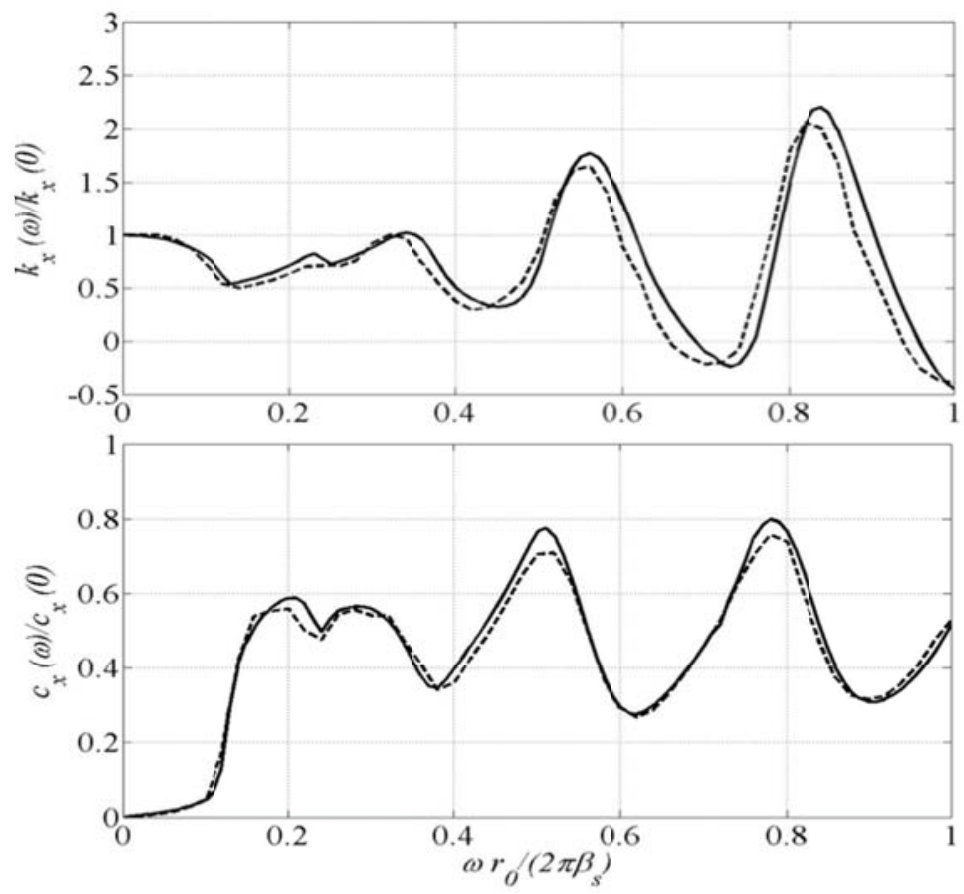

Figura 6. Funciones de impedancia normalizadas para un disco superficial rígido calculadas por Luco y Westman (línea punteada) y con la metodología propuesta (línea continua)

Se validó el método comparando con los resultados de Luco y Westman (1971) para un disco superficial rígido desplantado sobre un medio homogéneo de espesor finito que descansa sobre una base 
rígida. Los resultados para la parte real y la parte imaginaria de la función de impedancia se presentan en la figura 6. Las gráficas se encuentran normalizadas con respecto a los valores para frecuencia cero.

Estos resultados se reprodujeron con el método propuesto, considerando solamente que la zona rígida de la pila corresponde al primer nodo del sistema, teniendo así una pila de longitud cero. Se muestra en las gráficas que la reproducción de éstos resultados con el método propuesto es aceptable.

\section{ANÁLISIS Y RESULTADOS NUMÉRICOS}

Para estudiar la influencia de diferentes parámetros en la distribución de los elementos mecánicos producidos por acciones sísmicas en pilas, varios grupos de pruebas fueron diseñados. Los parámetros que se variaron son: la relación de esbeltez de la pila, la rigidez relativa entre la pila y el suelo y las propiedades del suelo. Se presentan gráficas normalizadas de la presión del suelo sobre la pila $\left(F_{0}\right)$, la fuerza cortante $\left(Q_{0}\right)$ y el momento flexionante $\left(M_{0}\right)$ en términos de la profundidad normalizada $(z / L)$.

Se estudiaron dos tipos de problemas:

- Con excitación en la base. A fin de caracterizar la influencia de la interacción cinemática, se analiza la respuesta armónica de la pila con cabeza fija, ante la incidencia vertical de ondas SH de amplitud unitaria. Las fuerzas de empuje del suelo y las fuerzas cortantes se normalizan con $\pi r_{0} \rho_{0} \ddot{x}_{g} L^{2}$ y los momentos flexionantes con $\pi r_{0} \rho_{0} \ddot{x}_{g} L^{3}$ siendo $L$ la longitud de la pila y $r_{0}$ el radio de la misma.

- Con excitación en la cabeza. Se estudia la respuesta de la pila bajo una excitación armónica unitaria en su cabeza para analizar la influencia de la interacción inercial en la respuesta de la pila. Tanto las presiones del suelo como las fuerzas cortantes y los momentos flexionantes se normalizan con respecto al valor máximo de fuerza cortante.

Vale la pena hacer notar que, mientras las solicitaciones en la cabeza de la pila, dependen casi exclusivamente de la respuesta dinámica de la superestructura, el movimiento de la excitación introducido en la base depende básicamente de la respuesta dinámica del suelo circundante. Es por esto que se puede decir que el caso de excitación en la base es dependiente de los desplazamientos del terreno, mientras que el caso de excitación en la cabeza es dependiente de las fuerzas introducidas en la cabeza de la pila. De ahí las diferentes normalizaciones para ambos casos.

Las variables utilizadas en el estudio son:

$\beta_{s} / \beta_{0}=$ relación entre la velocidad de ondas de cortante del suelo y de la pila

$\rho_{s} / \rho_{0}=$ relación entre la densidad del suelo y la pila

$v_{s}, v_{0}=$ relación de Poisson del suelo y de la pila

$\zeta_{s}, \zeta_{0}=$ amortiguamiento del suelo y de la pila

$L / r_{0}=$ relación de esbeltez de la pila 
Los parámetros utilizados en este análisis han sido considerados con la finalidad de representar los casos más comunes que se presentan en la práctica. Para las relaciones de esbeltez se utilizó un intervalo de valores similar al de estudios previos (Novak y El Sharnouby 1983). En cuanto a los contrastes de velocidades de onda de cortante se utilizaron valores que representen relaciones de $E_{0} / E_{s}$ desde 33 hasta 2000. Cruz et al en 2007 presentan un análisis de las relaciones $E_{0} / E_{s}$ que se presentan en tres distintos lugares representativos de México para pilotes de concreto. En su análisis muestra que los valores varían principalmente entre 40 y 1200.

La longitud activa de la pila es un parámetro que permite analizar de una mejor manera su comportamiento ante cargas laterales. La expresión para determinarla está dada por (Nikolau et al 2001).

$$
L_{a} \approx 1.5\left(\frac{E_{0}}{E_{s}}\right)^{1 / 4} d_{0}
$$

Si se sustituye la relación de esbeltez $L / r_{0}$ en la ecuación 1 para expresar la longitud activa en términos del porcentaje de la pila que se encuentra sujeta a esfuerzos $L_{a} / L$ se tiene que:

$$
\frac{L_{a}}{L} \approx 3.0\left(\frac{E_{0}}{E_{s}}\right)^{1 / 4} \frac{1}{\frac{L}{r_{0}}}
$$

En este estudio se consideró que la relación de Poisson de los materiales tanto de la pila como del suelo son iguales, por lo cual la ecuación 2 puede expresarse en términos de las velocidades de ondas de cortante como:

$$
\frac{L_{a}}{L} \approx 3.0\left(\frac{1}{\frac{\rho_{s} \beta_{s}^{2}}{\rho_{0} \beta_{0}^{2}}}\right)^{1 / 4} \frac{1}{L}
$$

Con esta expresión es posible definir el porcentaje de la longitud total de la pila $\left(L_{a} / L\right)$ que se encuentra sujeto a esfuerzos para distintas relaciones de esbeltez $\left(L / r_{0}\right)$.

\section{Pilas con distintas relaciones de esbeltez}

Para esta parte del análisis se consideraron los parámetros presentados en la tabla 2. Las variaciones de $F_{0}, Q_{0}$ y $M_{0}$ se presentan en la figura 7.

Se puede observar que la relación de esbeltez es un parámetro fundamental en la magnitud de la presión que ejerce el suelo sobre la pila, así como en la fuerza cortante y el momento flexionante que se desarrollan. Además de que afecta directamente la distribución de las presiones del suelo sobre la pila. 
Tabla 2. Parámetros para pilas con distintas relaciones de esbeltez

\begin{tabular}{ccccccc}
\hline Prueba & $\beta_{s} / \beta_{0}$ & $\rho_{s} / \rho_{0}$ & $v_{s}=v_{0}$ & $\zeta_{s}=\zeta_{0}$ & $L / r_{0}$ & $L_{a} / L$ \\
\hline A1 & 0.05 & 0.75 & 0.5 & 0.05 & 10 & 1.44 \\
A2 & 0.05 & 0.75 & 0.5 & 0.05 & 20 & 0.72 \\
A3 & 0.05 & 0.75 & 0.5 & 0.05 & 30 & 0.48 \\
A4 & 0.05 & 0.75 & 0.5 & 0.05 & 60 & 0.24 \\
A5 & 0.05 & 0.75 & 0.5 & 0.05 & 90 & 0.16 \\
\hline
\end{tabular}

En términos de la fuerza cortante y el momento flexionante para excitación en la base, se puede observar que mientras la relación de esbeltez se vuelve menor, la magnitud de los elementos mecánicos crece. La posición en la cual los elementos mecánicos se vuelven cero es muy parecida para todos los casos y en general la forma de la distribución es muy similar para todas las pilas. De aquí se puede demostrar que la relación de esbeltez es un parámetro determínate en el desempeño sísmico de las pilas.

Cuando se estudia el caso de excitación en la cabeza, el efecto de la relación de esbeltez es muy similar al de excitación en la base, ya que cuando la relación de esbeltez de la pila es más grande, la rigidez de la pila crece, produciendo que la fuerza necesaria para producir un desplazamiento unitario en la pila sea mayor. En cuanto a la forma de las curvas se puede observar que mientras la pila es más rígida, la longitud activa de la pila es mayor, como se muestra en la tabla 2. También es claro que mientras la pila es más rígida, la relación entre el momento flexionante máximo y la fuerza cortante máxima es mayor.

Es interesante ver que el máximo empuje del suelo sobre la pila no se presenta en la cabeza, su posición depende de la relación de esbeltez. Mientras la pila es más rígida, la posición del máximo empuje del suelo es más profunda, y la zona en la cual la pila se encuentra sujeta a presiones es mayor. La relación entre el máximo empuje del suelo y el cortante en la cabeza de la pila es mayor a medida que la pila es más esbelta.

La tabla 2 muestra que la pila con menor relación de esbeltez $\left(L / r_{0}=10\right)$ tiene un porcentaje de longitud activa mayor al $100 \%$, lo que significa que toda la pila se encuentra influida por las fuerzas que se desarrollen en la cabeza, condición que produce que para el caso de la excitación en la cabeza, toda la pila se encuentra trabajando.

Por otra parte, cuando se analiza la pila más esbelta $\left(L / r_{0}=90\right)$, se muestra que el porcentaje de longitud activa es de solo el $16 \%$, lo cual produce que cuando se somete a excitaciones en la cabeza, solo la parte superior de la pila se encuentra sometida a momento flexionante y fuerza cortante.

Es claro que a diferencia de las pilas sujetas a fuerzas en su cabeza, cuando se considera el caso del paso de las ondas sísmicas, la totalidad de la pila se encuentra sujeta solicitaciones mecánicas, independientemente de la relación de esbeltez de éstas, diferencia fundamental entre ambos fenómenos. 

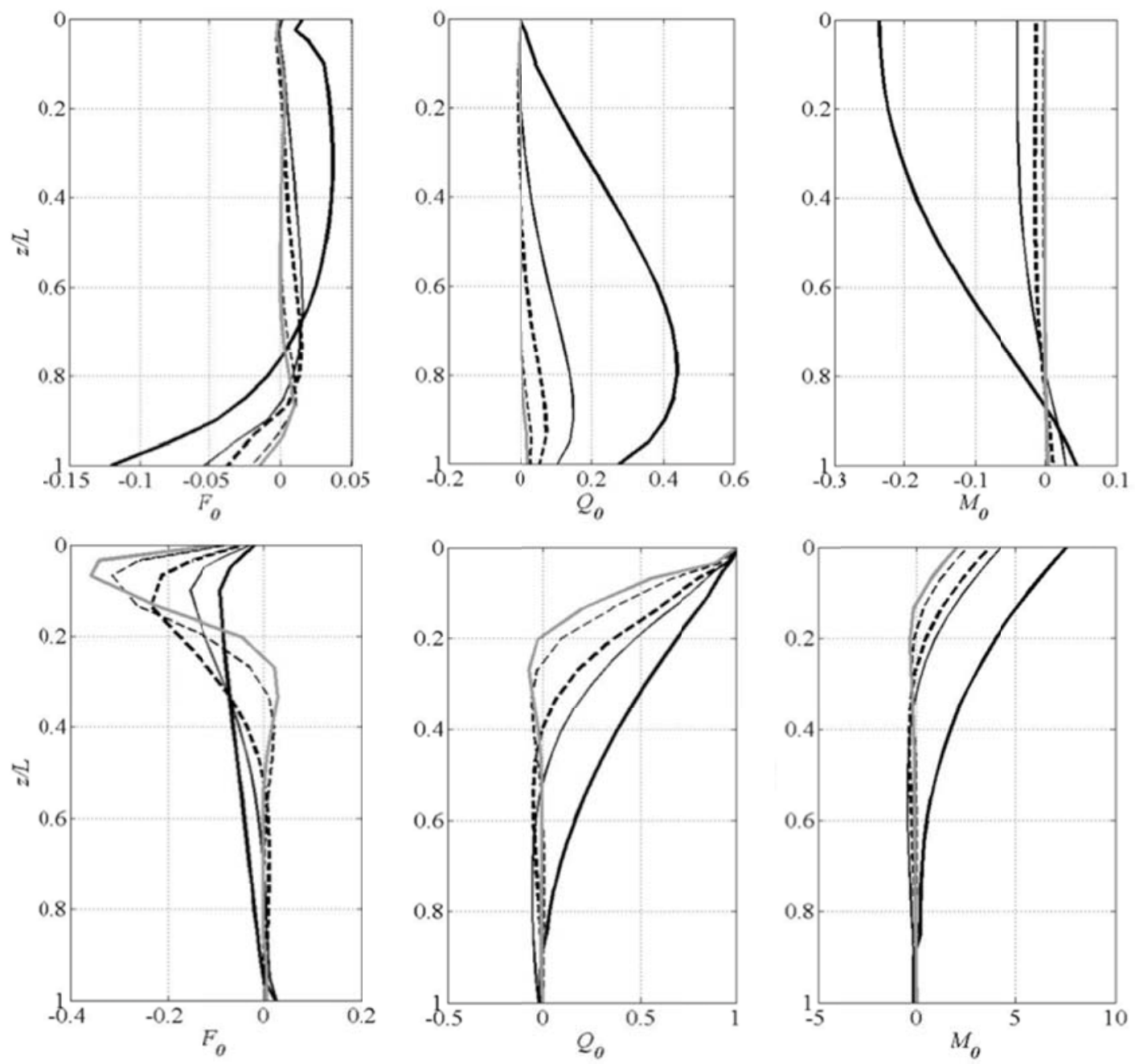

$-L / r_{0}=10-L / r_{0}=20--L / r_{0}=30--L / r_{0}=60-L / r_{0}=90$

Figura 7. Variación de $F_{0}, Q_{0}$ y $M_{0}$ para pilas con diferentes relaciones de $L / r_{0}$ para el caso del paso de ondas (superior) y excitación en la cabeza (inferior)

\section{Distintas relaciones de velocidad de onda de cortante entre el suelo y la pila}

Otro parámetro muy importante para el desempeño sísmico de las pilas es la velocidad de ondas de cortante del suelo y de la pila. Los parámetros que se utilizaron para esta parte del estudio se presentan en la tabla 3. Las curvas de $F_{0}, Q_{0}$ y $M_{0}$ se presentan en la figura 8.

En este grupo de pruebas sometidas a excitación en la base se demuestra que a medida que el contraste entre las velocidades de onda es mayor, las magnitudes de los elementos mecánicos crecen. Con este resultado se puede definir que el parámetro fundamental que controla la magnitud de los elementos mecánicos en las pilas introducidos por la interacción cinemática es la rigidez relativa entre la pila y el medio. Este parámetro queda definido por la relación de esbeltez del elemento y el contraste de velocidades de onda de cortante. 

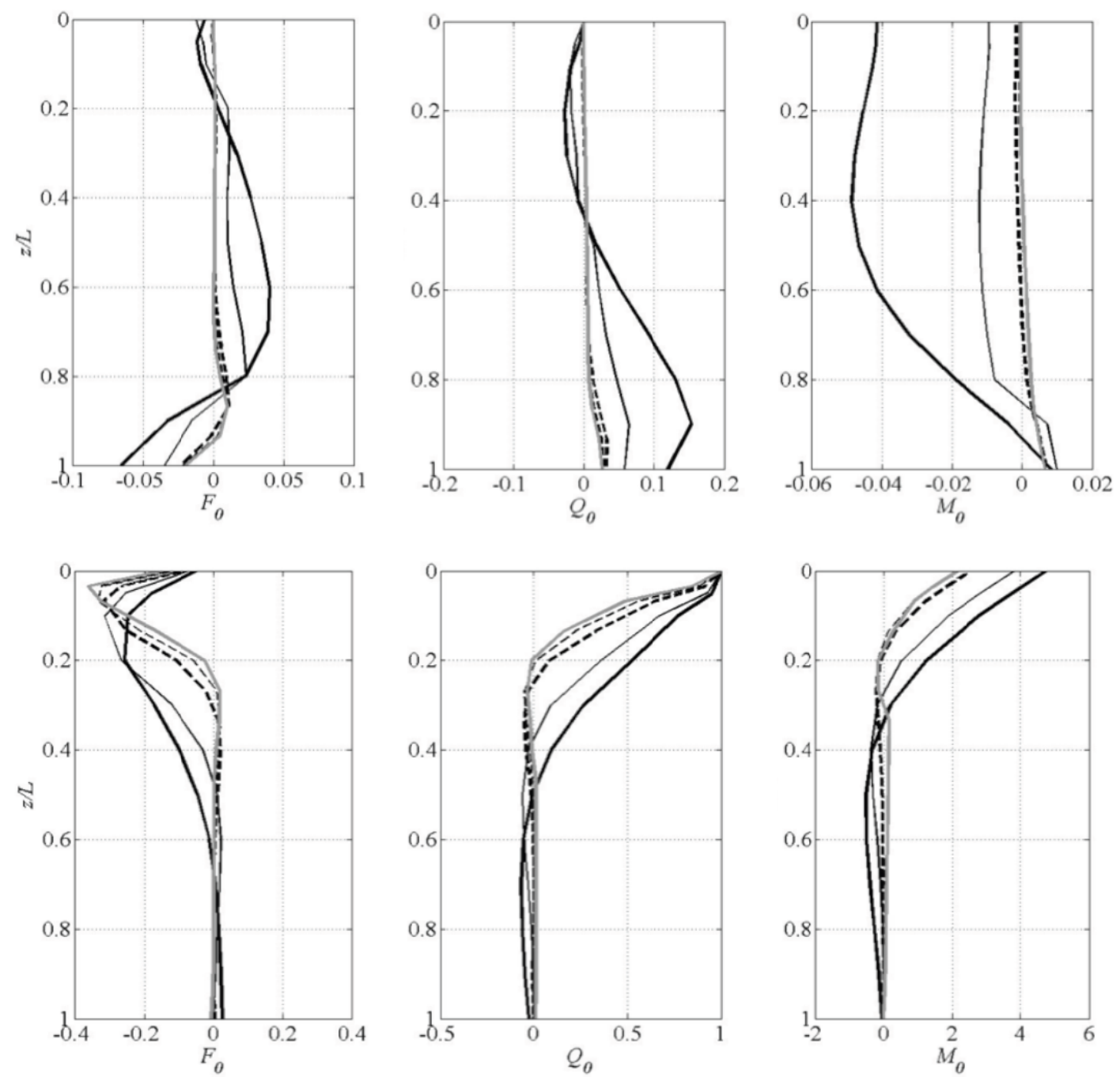

$-\beta_{s}^{\prime} \beta_{0}=0.025-\beta_{s}^{\prime} \beta_{0}=0.05---\beta_{s}^{\prime} \beta_{0}=0.10---\beta_{s}^{\prime} \beta_{0}=0.15-\beta_{s}^{\prime} \beta_{0}=0.20$

Figura 8. Variación de $F_{0}$, $Q_{0}$ y $M_{0}$ para diferentes relaciones de $\beta_{s} / \beta_{0}$ para el caso del paso de ondas (superior) y excitación en la cabeza (inferior)

Se puede observar que a medida que el contraste de velocidades de onda de cortante entre la pila y el suelo es mayor, la longitud activa de la pila crece como se muestra en la tabla 3 . De aquí se puede demostrar que también para el caso de excitación en la cabeza, el parámetro que controla el fenómeno es la rigidez relativa de la pila respecto al medio de desplante.

La longitud en la cual la pila está sujeta a empujes se vuelve más grande a medida que el contraste entre las velocidades de ondas de cortante del suelo y la pila es mayor. Nuevamente se observa que la relación entre el empuje máximo del suelo y el cortante máximo de la pila crece a medida que la pila es menos rígida con respecto al suelo. De igual forma se muestra que mientras la pila es más rígida con respecto al terreno, la relación entre el momento flexionante máximo y la fuerza cortante máxima crece. 
Tabla 3. Parámetros para pilas con distintas relaciones de velocidades de propagación de onda

\begin{tabular}{ccccccc}
\hline Prueba & $\beta_{s} / \beta_{0}$ & $\rho_{s} / \rho_{0}$ & $v_{s}=v_{0}$ & $\zeta_{s}=\zeta_{0}$ & $L / r_{0}$ & $L_{a} / L$ \\
\hline B1 & 0.025 & 0.75 & 0.5 & 0.05 & 40 & 0.51 \\
B2 & 0.05 & 0.75 & 0.5 & 0.05 & 40 & 0.36 \\
B3 & 0.10 & 0.75 & 0.5 & 0.05 & 40 & 0.25 \\
B4 & 0.15 & 0.75 & 0.5 & 0.05 & 40 & 0.21 \\
B5 & 0.20 & 0.75 & 0.5 & 0.05 & 40 & 0.18 \\
\hline
\end{tabular}

La principal diferencia respecto al caso de excitación en la base es que mientras en las pilas sujetas a excitación en la cabeza la variación se presentan primordialmente en la longitud activa, para el caso de la interacción cinemática, la influencia de la rigidez relativa se expresa en términos de la magnitud de las fuerzas. Cuando se considera la interacción cinemática, la totalidad de la pila se encuentra sujeta a fuerzas internas. Este punto es muy importante, ya que si solo se considera la influencia de las cargas que se presentan en la cabeza de la pila en el diseño, se puede menospreciar el valor de las fuerzas que se desarrollarán en las zonas más profundas de la pila, sobre todo en lugares por debajo de la longitud activa.

\section{Medios estratificados}

La presencia de estratos con distinta rigidez tiene una importante influencia en la distribución de los elementos mecánicos en las pilas producido por el paso de ondas sísmicas. Para evaluar dicha influencia, se estudia un medio compuesto de dos estratos. El estrato superior tiene un espesor de $L / 2$. Las propiedades del estrato superior e inferior se denotan con los subíndices 1 y 2, respectivamente. Ambos estratos tienen las mismas propiedades excepto por la velocidad de propagación de onda. Los parámetros utilizados se presentan en la tabla 4. Las curvas de $F_{0}, Q_{0}$ y $M_{0}$ se presentan en la figura 9.

Tabla 4. Parámetros para pilas en un medio con dos estratos

\begin{tabular}{cccccccc}
\hline Prueba & $\beta_{\text {s1 }} / \beta_{0}$ & $\rho_{\text {s1 }} / \rho_{0}$ & $\rho_{\text {s2 }} / \rho_{0}$ & $v_{\text {s1 }}=v_{\mathrm{s} 2}=v_{0}$ & $\zeta_{\mathrm{s} 1}=\zeta_{\mathrm{s} 2}=\zeta_{0}$ & $L / r_{0}$ & $\beta_{\mathrm{s} 1} / \beta_{\mathrm{s} 2}$ \\
\hline C1 & 0.025 & 0.75 & 0.75 & 0.5 & 0.05 & 40 & 1.00 \\
C2 & 0.025 & 0.75 & 0.75 & 0.5 & 0.05 & 40 & 0.50 \\
C3 & 0.025 & 0.75 & 0.75 & 0.5 & 0.05 & 40 & 0.25 \\
C4 & 0.025 & 0.75 & 0.75 & 0.5 & 0.05 & 40 & 0.10 \\
\hline
\end{tabular}

Se puede ver que cuando se tiene un medio estratificado sometido al paso de ondas sísmicas, existe una concentración de fuerzas a la profundidad de la interfaz $(z / L=0.5)$, y la magnitud de esta fuerza crece a medida que el contraste de velocidades de onda de cortante entre los estratos es mayor. Este efecto de concentración de fuerza en la interfaz ha sido previamente identificado por otros autores (Nikolau et al 2001, Poulos 2007, Dezi et al 2010). Para el caso de un medio homogéneo $\left(\beta_{\mathrm{s} 1} / \beta_{\mathrm{s} 2}=1.0\right)$ es claro que no existe dicha concentración de fuerzas a la profundidad de la interfaz. 

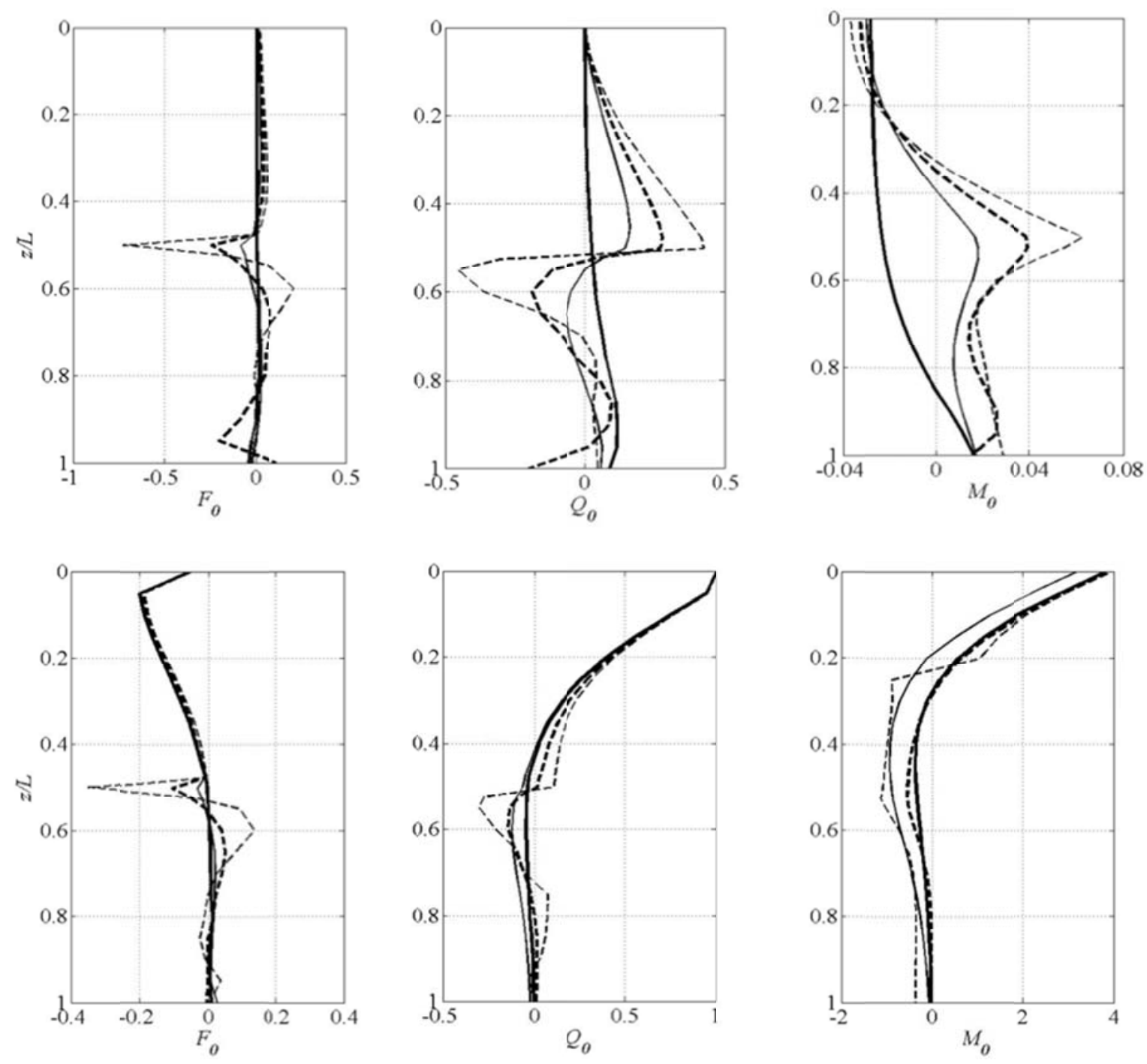

$-\beta_{s 1} / \beta_{s 2}=1.0-\beta_{s 1} / \beta_{s 2}=0.5---\beta_{s 1} / \beta_{s 2}=0.25---\beta_{s 1} / \beta_{s 2}=0.10$

Figura 9. Variación de $F_{0}, Q_{0}$ y $M_{0}$ para para medios estratificados con distintos contrastes de $\beta_{\mathrm{s} 1} / \beta_{\mathrm{s} 2}$ para el caso del paso de ondas (superior) y excitación en la cabeza (inferior).

Cuando se considera el caso de excitación en la cabeza, se puede observar que las curvas son muy similares para todos los casos, con una pequeña diferencia a la profundidad de la interfaz, en donde se manifiesta una concentración de empuje del suelo. La magnitud de este incremento es pequeña con relación a la magnitud de las fuerzas en la cabeza. Para el caso de mayor contraste de rigideces entre los estratos $\left(\beta_{\mathrm{s} 1} / \beta_{\mathrm{s} 2}=0.1\right.$ ), la concentración de la fuerza cortante llega a ser de alrededor del $25 \%$ de la fuerza cortante máxima, lo que manifiesta que incluso para excitación en la cabeza, si la longitud activa de la pila es mayor que el espesor del estrato superior, el efecto de la presencia de dos medios con distinta rigidez puede ser importante. Es interesante que para este caso, la influencia del estrato más profundo es mínima en relación con el caso de excitación en la base. 
Este efecto se puede observar fácilmente en las curvas de empujes del suelo, en donde a la profundidad de la interfaz se muestra una concentración para ambos casos; sin embargo para el caso de excitación en la cabeza, solo en la pila con el mayor contraste de rigideces entre los estratos ( $\beta_{s 1} / \beta_{s 2}=0.1$ ), el máximo empuje del suelo corresponde a esta profundidad.

Debido a la poca influencia que tiene en general en el comportamiento lateral de las pilas sujetas a cargas en la cabeza, el efecto de la estratificación en la distribución de los elementos mecánicos no es considerado en los análisis convencionales. Es claro que para el caso del paso de ondas sísmicas esta condición puede producir grandes concentraciones de esfuerzo en la interfaz.

\section{Diferentes condiciones de apoyo}

Un caso muy común en medios estratificados, es el uso de pilas cuya punta se encuentra embebida en un estrato con una rigidez mayor, con la finalidad de asegurar una alta restricción rotacional en la punta. Para estudiar el efecto de la condición de apoyo de la punta en los elementos mecánicos que desarrollará la pila, se llevó a cabo el siguiente análisis.

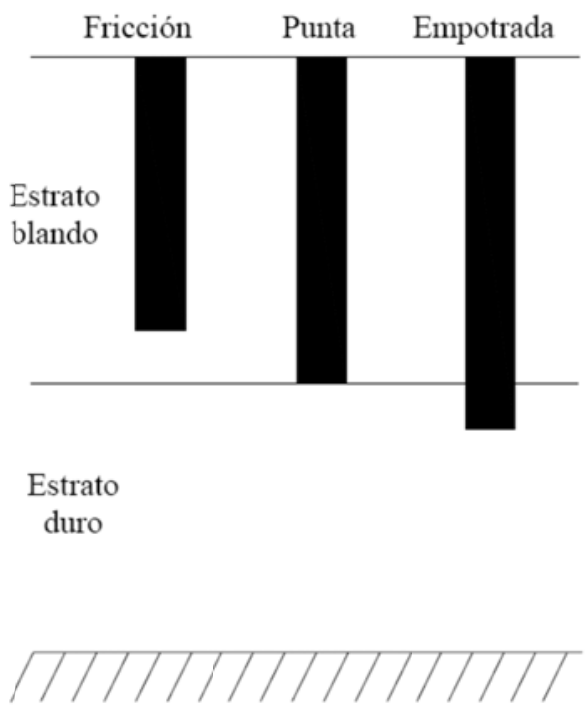

Figura 10. Esquema de las pilas de fricción, de punta y empotrada

Se definen tres diferentes pilas, desplantadas en un medio compuesto por dos estratos. Los parámetros utilizados se muestran en la tabla 5.

Todas las pilas tienen el mismo radio $\left(r_{0}\right)$. El estrato superior tiene un espesor equivalente a $40 r_{0}, \mathrm{y}$ el estrato inferior un espesor correspondiente a $120 r_{0}$, este espesor esta dado con la finalidad de alejar la base infinitamente rígida lo suficiente para evitar problemas de frontera en el modelo numérico. El perfil estratigráfico se ha definido en relación con el subsuelo de la ciudad de México, en donde una arcilla muy blanda ( $\beta_{\mathrm{s}}=60 \mathrm{~m} / \mathrm{s}$ ) se encuentra sobre un estrato más rígido ( $\beta_{\mathrm{s}}=250-300 \mathrm{~m} / \mathrm{s}$ ) (Ovando 1996). Se toma el caso del subsuelo de la ciudad de México por ser un referente de suelos blandos en el mundo. 

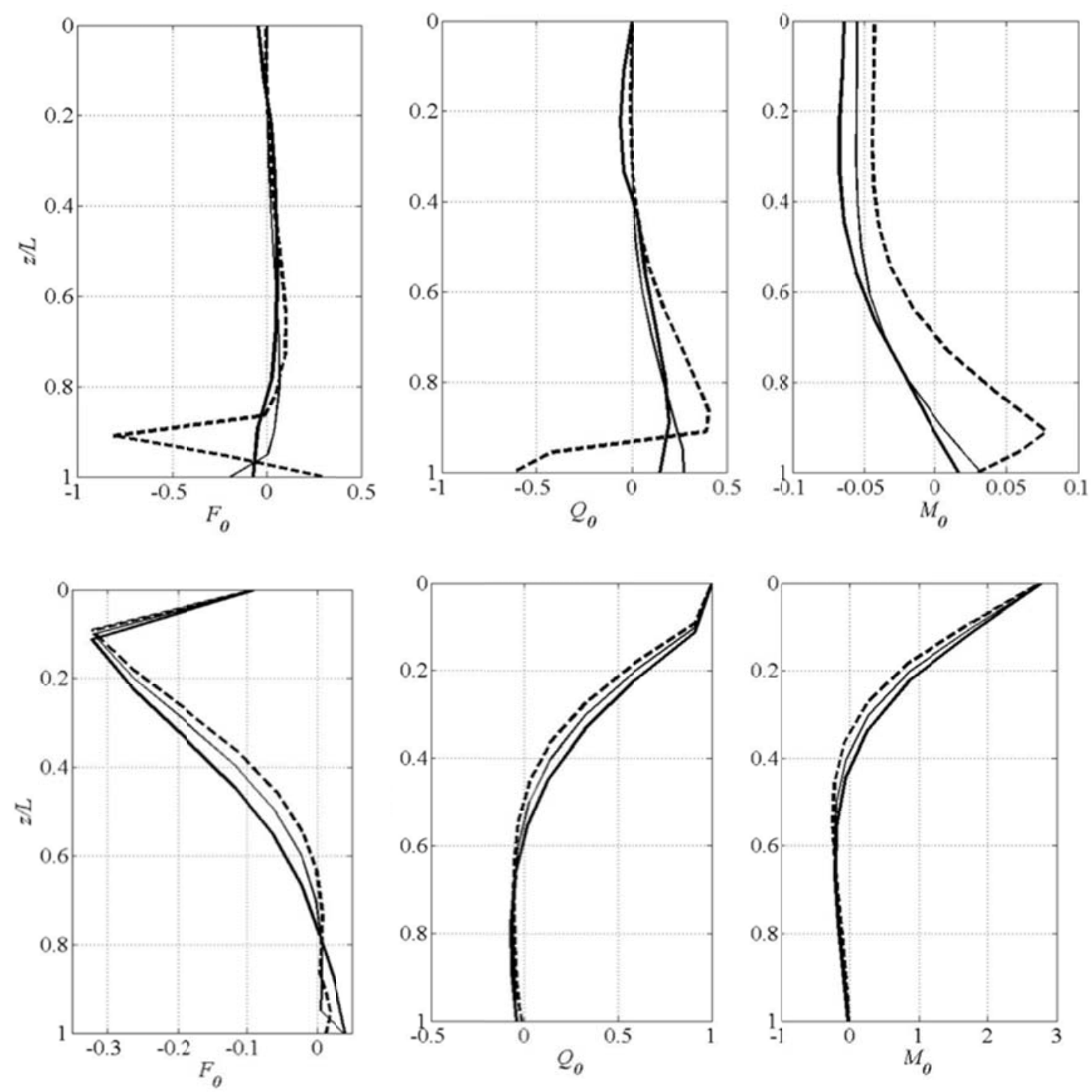

\section{Fricción - Punta ---Empotrada}

Figura 11. Variación de $F_{0}, Q_{0}$ y $M_{0}$ para pilas con distintas condiciones de apoyo en la punta para el caso del paso de ondas (superior) y excitación en la cabeza (inferior)

Tabla 5. Parámetros para pilas en con distintas condiciones de apoyo

\begin{tabular}{ccccccc}
\hline$\beta_{\mathrm{s} 1} / \beta_{0}$ & $\rho_{\mathrm{s} 1} / \rho_{0}$ & $\rho_{\mathrm{s} 2} / \rho_{0}$ & $v_{\mathrm{s} 1}=v_{\mathrm{s} 2}=v_{0}$ & $\zeta_{\mathrm{s} 1}=\zeta_{\mathrm{s} 2}=\zeta_{0}$ & $L / r_{0}$ & $\beta_{\mathrm{s} 1} / \beta_{\mathrm{s} 2}$ \\
\hline 0.025 & 0.75 & 0.75 & 0.5 & 0.05 & 40 & 0.25 \\
\hline
\end{tabular}

La diferencia entre las pilas es la condición de apoyo de la punta. Se consideró una pila de fricción, una pila de punta y una pila empotrada en la punta. La pila de fricción corresponde a una pila cuya punta 
se encuentra dentro del estrato blando ( $L=36 r_{0}$ ), en la pila de punta se considera que la punta se encuentra simplemente apoyada sobre el estrato duro, sin tener ninguna longitud de empotre $\left(L=40 r_{0}\right)$ y la pila empotrada corresponde a una pila cuya punta se encuentra embebida en el estrato duro ( $\left.L=44 r_{0}\right)$. La geometría de las tres pilas se presenta en la figura 10. Las curvas de $F_{0}, Q_{0}$ y $M_{0}$ se presentan en la figura 11 .

Para el paso de las ondas sísmicas, todas las pilas manifiestan un comportamiento similar en términos de los empujes del suelo en casi la totalidad de la longitud. La única diferencia se encuentra a la profundidad de desplante de las pilas. Para la pila de punta existe un cierto incremento en el empuje del suelo en relación con la pila de fricción, sin embargo para la pila empotrada el incremento es significativamente mayor.

Este incremento en los empujes de suelo afecta tanto la fuerza cortante como el momento flexionante. Existe un aumento significativo de ambos elementos mecánicos en la profundidad de la interfaz. Este es un efecto muy importante ya que prueba que cuando se empotra una pila con la finalidad de tener un mejor trabajo geotécnico, ésta puede desarrollar elementos mecánicos de gran magnitud.

Por otra parte, cuando se analiza la excitación en la cabeza, se muestra claramente que la influencia de la condición de apoyo de la punta no es muy importante. En la figura 11 se muestra que la distribución de empujes del suelo, la fuerza cortante y el momento flexionante no se ven influidos por la condición de apoyo, a diferencia del caso de excitación en la base.

De aquí se puede concluir que dicho parámetro no tiene mucha influencia en el comportamiento lateral de pilas cargadas en su cabeza, mientras que para el caso del paso de ondas sísmicas es de gran importancia.

\section{Estrato equivalente}

En la mayoría de los suelos se presenta un incremento de la rigidez en relación con la profundidad. Para algunas arcillas comunes, este incremento tiene un comportamiento casi lineal, como se muestra en los resultados presentados por Ovando (1996).

Con la finalidad de simplificar los problemas, es común analizar el comportamiento de pilas desplantadas en medios estratificados, con un medio homogéneo equivalente, considerando propiedades equivalentes de los estratos presentes en el problema. Estas propiedades pueden determinarse con distintos procedimientos como el propuesto por Gómez en 2000.

Para establecer la influencia de esta simplificación en la distribución de los elementos mecánicos que desarrollarán las pilas cargadas lateralmente, se realiza la comparación de una pila desplantada en un medio heterogéneo con un incremento lineal de la velocidad de propagación de ondas, y la misma pila desplantada en un medio homogéneo equivalente.

La pila tiene una relación de esbeltez $L / r_{0}=40$. El medio estratificado tiene una variación de la velocidad de propagación de ondas de cortante $\left(\beta_{\mathrm{s}}\right)$ de $35 \mathrm{~m} / \mathrm{s}$ a $100 \mathrm{~m} / \mathrm{s}$ en la longitud de la pila. El medio equivalente tiene una velocidad de propagación $\left(\beta_{\mathrm{s}}\right.$ ) de $65 \mathrm{~m} / \mathrm{s}$ y un espesor igual a la longitud de la pila. Las variaciones de $F_{0}, Q_{0}$ y $M_{0}$ se muestran en la figura 12. Es claro que considerar un estrato equivalente produce una variación en la distribución de los empujes del suelo, la fuerza cortante y el momento flexionante ante el paso de ondas sísmicas. La forma de las curvas también cambia. 

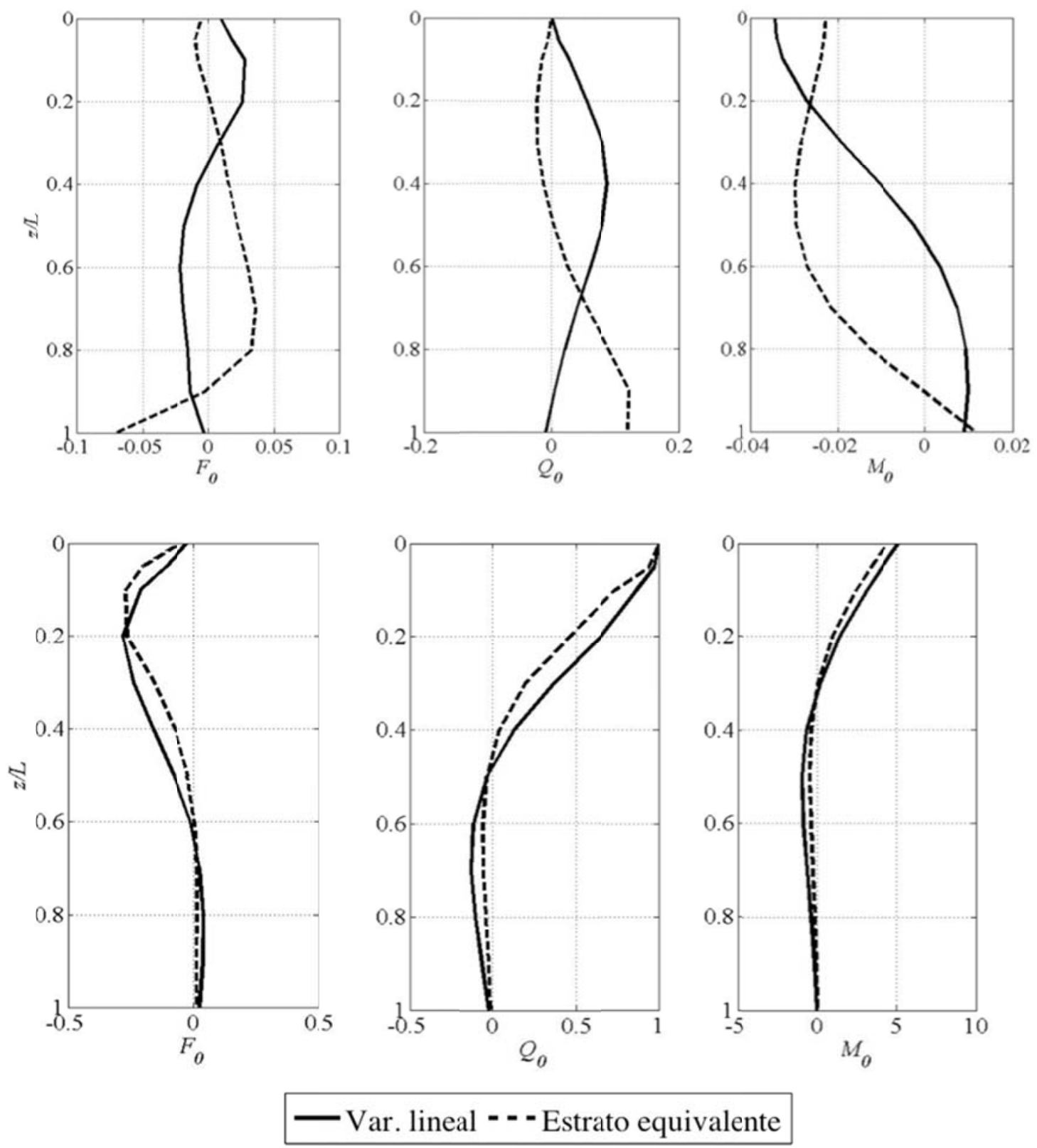

Figura 12. Variación de $F_{0}, Q_{0}$ y $M_{0}$ para una pila en un medio heterogéneo y en un estrato homogéneo equivalente para el caso del paso de ondas (superior) y excitación en la cabeza (inferior)

Para la excitación en la base el máximo momento flexionante para el estrato equivalente se presenta a la mitad de la pila, mientras que para el medio heterogéneo el valor de momento máximo se presenta en la cabeza de la pila.

Se muestra que la influencia de considerar un estrato equivalente es mínima para la distribución de los empujes del suelo, la fuerza cortante y el momento flexionante cuando se consideran fuerzas en la cabeza de la pila. La única diferencia radica en que en el estrato equivalente se manifiesta una longitud activa de la pila menor que para el caso del medio heterogéneo, debido a que los estratos superiores presentan una rigidez menor que la del estrato equivalente. 
Debido a la poca influencia que tiene esta simplificación cuando se considera que la pila está sujeta a fuerzas en su cabeza, se ha vuelto muy común en el análisis y diseño de pilas cargadas lateralmente, sin embargo parece inadecuada cuando se analiza el trabajo de la pila ante la incidencia de ondas sísmicas.

\section{COMENTARIOS Y CONCLUSIONES}

En el presente trabajo se utilizó un modelo para analizar el comportamiento de pilas bajo acciones laterales y los elementos mecánicos que se desarrollan en ellas, con la posibilidad de considerar distintos escenarios.

Se comparó la metodología con otros modelos de interacción cinemática (Nikolau et al 2001), y se obtuvieron resultados aceptables, con diferencias en la punta de las pilas, en donde en general los métodos simplificados predicen un trabajo nulo del elemento, mientras que la metodología propuesta manifiesta que en realidad existe cierto valor de momento flexionante.

Tanto para la interacción cinemática como para la inercial se comprobó que a medida que la pila es más robusta desarrolla elementos mecánicos mayores. Para el caso de excitación en la cabeza, se mostró que a medida que la pila es más esbelta, la relación entre el máximo empuje del suelo y la máxima fuerza cortante crece. Variando también el contraste de rigideces entre el material de la pila y el suelo, se concluyó que a medida que el contraste de rigideces es más drástico, y por lo tanto la diferencia en los desplazamientos en ambos medios, los elementos mecánicos asociados con la interacción cinemática crecen. Para la excitación en la cabeza de la pila, se observó que este parámetro tiene influencia en la longitud activa de la misma. Además, la relación entre el máximo empuje que ejerce el suelo sobre la pila y la máxima fuerza cortante crece a medida que el contraste de velocidades de onda es menor. Con esto, se corroboró que la forma de la distribución de los elementos mecánicos en la pilas varía básicamente con la rigidez relativa de la pila respecto a la rigidez del suelo.

También se analizó la presencia de medios estratificados, mostrando que este parámetro tiene una gran importancia cuando se considera la interacción cinemática. Se ratificó que el contraste de rigidez entre dos estratos produce concentraciones de elementos mecánicos justo en la interfaz de ellos. Esta concentración crece a medida que el contraste de velocidades de propagación de ondas de cortante es mayor. Para el caso más drástico $\left(\beta_{\mathrm{s} 1} / \beta_{\mathrm{s} 2}=0.1\right)$, la relación entre el momento en la interfaz y en la cabeza es de 1.5. Dicho fenómeno se manifiesta claramente en las curvas de empuje lateral del suelo. Cuando se consideró la excitación en la cabeza de la pila, se prueba que si la longitud activa es menor que el espesor del estrato superior, la influencia de la presencia de estratos con distintas propiedades no es significativa. Si la longitud activa llega a ser mayor que el espesor del estrato superior, y el contraste de velocidades de propagación de ondas de cortante es grande, la concentración de fuerza debida a la presencia de estratos con distintas propiedades es importante, por ejemplo para el caso de $\beta_{s 1} / \beta_{s 2}=0.1$ la fuerza cortante y el momento flexionante en la interfaz son del orden del $25 \%$ de los elementos producidos en la cabeza. Este efecto puede producir fallas si no es considerado correctamente. Además, la estratigrafía influye de manera notable en la distribución y magnitud de las fuerzas.

Otro factor que se caracteriza en este trabajo es la condición de apoyo de la punta. Se mostró que el incremento de los elementos mecánicos producidos por la interacción cinemática es notable cuando la pila se encuentra embebida en un estrato duro, debido al contraste de velocidades de propagación de onda de ambos estratos. Para el caso de excitación en la base y en que la pila no se encuentre en contacto directo con el medio de mayor rigidez, los elementos mecánicos para la parte inferior de la pila se reducen drásticamente, situación que debe tomarse en cuenta para el diseño de pilas de punta. El cortante de la pila 
de fricción en la punta corresponde solamente al $40 \%$ del de la pila con la punta embebida en el estrato más rígido, mientras que el momento flexionante corresponde solamente al $20 \%$ del de la pila con la mayor restricción en la punta. Por otra parte se observó que cuando se consideran fuerzas en la cabeza de la pila, la condición de apoyo de la punta no tiene mucha influencia en el trabajo de ésta ante cargas laterales. Cuando se considera el paso de las ondas sísmicas, se observó que utilizar un medio homogéneo equivalente para analizar medios estratificados, produce diferencias en la respuesta de la pila. Aunque el valor del cortante y el momento flexionante máximo no se incrementa, la distribución es completamente distinta. Por otro lado, cuando se considera la interacción inercial, la influencia de utilizar estratos equivalentes es mínima.

Con este estudio se puede observar que existen efectos que tienen un impacto mínimo cuando se considera que la pila está sujeta a fuerzas en su cabeza. En el caso de la interacción cinemática se observó que estas condiciones incrementan de manera importante los elementos mecánicos que se desarrollan en este tipo de cimentaciones.

Todos los resultados presentados en este trabajo se refieren al comportamiento elástico de los materiales. Para incluirse el comportamiento no lineal de manera aproximada, se consideran valores de los parámetros del suelo compatibles con los niveles de deformación esperados. Esto se traduce en un incremento en el contraste de rigideces pila-suelo.

\section{AGRADECIMIENTOS}

El primer autor agradece a la Coordinación de Estudios de Posgrado de la UNAM (CEP-UNAM) y al Instituto de Ingeniería de la UNAM (II-UNAM) por financiar la investigación doctoral de la cual se desprende el presente trabajo.

\section{REFERENCIAS}

Avilés López, J. y L. E. Pérez-Rocha, (2011) Presiones dinámicas del suelo en lumbreras, Revista de Ingeniería Sísmica, No 85, pp. 1-31.

Cruz C. y D. Murià (2007), Procedimiento simplificado para calcular rigideces y amortiguamientos en edificios cimentados con pilotes de fricción, tesis de maestría, Instituto de Ingeniería, UNAM, México, 139 pp.

Dezi F., S. Carbonari y G. Leoni. (2010a), Kinematic bending moments in pile foundations, Soil Dynamic and Earthquake Engineering, Vol. 30, pp. 119-132.

Fernández Sola L. R., J. Avilés-López y D. Murià Vila. (2011), Respuesta dinámica de pilas y pilotes de punta y de fricción ante cargas laterales, Tesis de doctorado, Instituto de ingeniería, UNAM, México, 95 pp.

Fernández Sola L.R., J. Avilés López y D. Murià Vila. (2012), Fully and partially toe restrained piles subjected to ground motion excitation, Soil Dynamics and Earthquake Engineering, aceptado para su publicación.

Gómez, A. (2000) Presentación de un modelo matemático para el cálculo del periodo fundamental de vibración en suelos estratificados con capacidad de predecir efectos de amplificación o atenuación dinámica, XII Congreso Nacional de Ingeniería Estructural, Sociedad Mexicana de Ingeniería Estructural, León, Guanajuato, México. 
Hall, W.S. (1994), The boundary element method, Solid Mechanics and its Applications, Vol. 27, pp. 6183.

Hashash, Y., J. Hook, B. Schmidt y J. Yao. (2001), Seismic design and analysis of underground structures, Tunneling and Underground Space Technology, Vol. 16, No. 4, pp. 247-293.

Luco, J.E. y R.A. Westmann. (1971), Dynamic response of circular footings, Journal of Engineering Mechanics Division, Vol. 97, (EM5), pp. 1381.

Lysmer J. y L. Drake. (1972), A finite element method for seismology”, Methods in Computational Physics. Advances in Research and Applications, Vol. 11, Seismology: Surface Waves and Earth Oscillations, Academic Press, California.

Mizuno, H. (1987), Pile Damage during earthquakes in Japan, Dynamic Response of Pile Foundations, ASCE Special Publication, pp. 53-78.

Nikolau, S., G. Mylonakys, G. Gazetas y T. Tazoh. (2001), Kinematic pile bending during earthquakes: analysis and field measurements, Geotechnique Vol. 51, No. 5,pp. 425-440.

Novak, M. y B. El Sharnouby. (1983) Stiffness constants of single piles, Journal of the Geotechnical and Engineering Division, Vol. 109, No. 7, pp. 961-974

Okamoto, S., C. Tamura, K. Kato y M. Hamada. (1973). Behaviors of submerged tunnels during earthquakes. Proceedings of the 5th World Conference on Earthquake Engineering, Roma, Italia, pp. 544-553.

Ovando, E. (1996) Initial dynamic stiffness of Mexico city clay from field test, Proceedings of the 11th World Conference on Earthquake Engineering, Acapulco, Guerrero, México.

Poulos, H.G. (2007), Ground movements - A hidden source of loading on deep foundations, Deep Foundations Institute Journal, Vol 1, No.1, pp. 37-53.

Tassoulas, J.L. (1981) Elements for the numerical analysis of wave motion in layered media, Massachusetts Institute of Technology Dept of Civil Engineering, Research Report R81-2, Order No. 689,

Tassoulas, J.L. y E. Kausel. (1983), Elements for the numerical analysis of wave motion in layered media, International Journal for Numerical Methods in Engineering, Vol. 19, pp. 1005-1032.

Veletsos, A.S. y A.H. Younan. (1995) Dynamic modeling and response of rigid embedded cylinders, Journal of Engineering Mechanics, Vol. 121, pp. 1026-1035.

Zienkiewicz, O. C. (1994), The finite element method, University of California Berkley, Vol. 1, 4ta edición, McGraw Hill 